\title{
Design and Implementation of Multimedia Material Library Based on Campus Network
}

\author{
Chenying Yang \\ The Chinese People’s Armed Police Forces Academy, Lang fang, China \\ y_feiruth@sina.com
}

\begin{abstract}
This study focuses on the extensive application of multimedia courseware in practical teaching and develops a set of multimedia material library based on campus network, according to the design and preparation of course material by the scholars. Besides, it introduces the significance of system development to teachers and students in the school, and sets forth the content, architecture, detailed design and the implementation of system design.
\end{abstract}

Keywords- multimedia; material Library; campus network

\section{BACKGROUND INTRODUCTION}

With the development of computer technology, computer-aided teaching and multimedia courseware are becoming increasingly popular. Multimedia content can make teaching more intuitive, easy to understand. Currently, most of the teachers have capacity in design and production of multimedia courseware, collecting and downloading of information from network; in addition, some students learn some courses and participate in the multimedia courseware competition. The courseware is varied and creative, and requires a large number of multimedia materials. However, it takes much time and wastes lots of resources for the courseware if the materials should be re-searched and remade ${ }^{[1]}$. It will be more convenient for teachers and students to use these materials if the multimedia materials can be stored or managed in a certain way and the material library can be established. Thus, it saves a lot of time and energy to make courseware ${ }^{[2]}$. Besides, teachers can also share the resources in the material library. Due to the school features, it is inconvenient for the teachers and students in our school to download materials. Instead, they download a large amount of data teaching materials on the campus network with high transmission speed. Thus, we develop the management system of multimedia materials for teachers and students based on campus network, for the convenience of browsing, inquiry, and downloading resources.

\section{Design Content AND DEVELOPMENT FrameWORK}

\section{A. Design Content of System}

The multimedia material library is developed according to the design and preparation of courses related to the major of electronic information. In the material library, the materials of classroom teaching is made into the forms of images, audio, video, etc. It mainly oriented by the teachers and students. In this design, it is important for the users to search the needed materials quickly according to the type of material. All the materials should be previewed online and downloaded. In order to keep the material constantly updated, the users are allowed to upload the collected or processed new material into the server. Thus, good resources can be shared to more users. For administrators, the administrator has the right to browse, modify and delete the data in the multimedia material library only if he logs in legally.

\section{B. Development Framework of System}

The designed system employs the B/S structural model while the system architecture takes the hierarchical design, thus improving the security of the system and reducing the development and maintenance costs effectively. As a result, the changing needs for the business can be met.

The system can be divided into the following aspects, including user interface, data buffering, database, and output display, etc.

The level of user interface is the closest to the user. It displays graphical interface for the users, for the convenience of basic operations for users. In the level, the users are entitled to input data, modify data, and delete data, set the optional standard, and set query conditions, and so on. In a word, all the operation of users can be done in the user interface.

The level of data buffering creates a temporary buffering area to save the uncommitted data. During the process, all the operations are completed by the system, including adding information in data buffer, information modification, removal of information, matching process, judgment of conditions legality, etc. During the process, system mainly carries out the work before submission, to ensure the legality and accuracy of submitted data and reduce database errors.

The level of database provides the room for operations in the database. The data in the data buffer can be stored in the database, and the query statement can be executed. The illegal operations and errors of the database can be reduced significantly, because the buffer has been checked in advance. Besides, abnormal situations can be cut back in query.

The output level shows the results to the users, including the query results, charts, error messages, messages, etc.

\section{Detailed DESIgn OF System}

Based on the campus network, the multimedia materials can be integrated, stored, and managed, and the database management system is established. It provides various functions, including the browsing and retrieval of materials, 


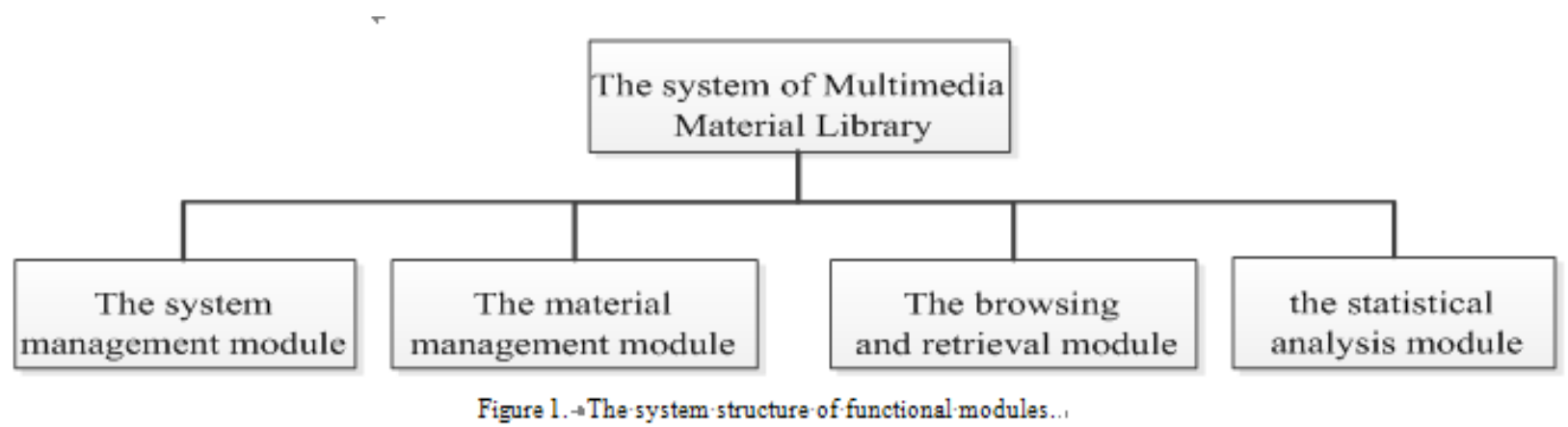

The system management module is composed of user management, rights management, log management and security management, etc. Various functions can be achieved, including the users adding and deleting, modification of the basic information, statistics of login times, and permissions assignment, etc. The authentication and authorization management have been carried out by means of the login module of user, in order to enhance the system security. If the user name is input, the system will identify the user ID in line with the data in the table automatically. Then the appropriate permissions can be assigned and the user is entitled to use.

The material management module mainly manages the material in the format of image, video, animation, audio, document, etc. Based on the material management module, various materials can be integrated, stored and managed. In addition, the materials can be queried and displayed according to the selections of user and the input keywords. It is also available to import or export the material.

The browsing and retrieval module and the statistical analysis module can be used for the searching of material resources. The user can browse and inquiry the stored material as long as he log in. the tree list in the left makes it more convenient to browse the material category while the tree list in the right displays the thumbnails. The main view displays the material as long as it is selected.

As for the material inquiry, the user is able to make accurate single query or fuzzy single query according to the name of models or components, ID No. or classification. In addition, the combined query with multiple conditions is available. During the process of the material inquiry, the query command is accepted by means of query conditions; the keywords are inquired through database interface; the features can be extracted by the method on the basis of keywords; the material resources can be searched in the material library depending on the established index. Finally, the results will be returned to the user.

\section{REALIZATION OF MAIN FUNCTIONS IN THE SYSTEM}

\section{A. Retrieval function}

Due to a wide range of multimedia material, it is difficult to find out the required resources. The system introduces two search methods, namely keyword retrieval and treeview retrieval ${ }^{[4]}$. The keyword retrieval requires the user to enter the name of the material for retrieval. Different data tables can be retrieved in line with the classification and keyword. The multimedia information resources, in the form of video, audio or images, can be searched by means of keyword retrieval. The searched results are practicable for previewing and downloading, more efficient and accurate for the location in the corresponding material. It results in high accuracy and completeness. Another retrieval method called tree-view retrieval is similar to the multi-level folders in the resource manager of windows, as well as the tree structure of files. The user searches the needed content through multilevel, fast and efficient. Thus, a lot of teaching information can be collected. All the information related to the query content is retrieved. The method is suitable for the materials in the same subject.

\section{B. Function of uploading materials}

The multimedia library is available for the users to upload material resources. As more users take part in, a more powerful multimedia resources database will be established. The resource uploading is related to the reception of form information and storage of uploaded files. In the resources upload interface, the users are required to fill in the resource name, keyword description, and resource type, etc. The main code is as follows: string TableName = "TB_MultiMedia_Material"; string fileSavePath = "res/materials/"; string file = "';

if (FileUpload1.HasFile) \{

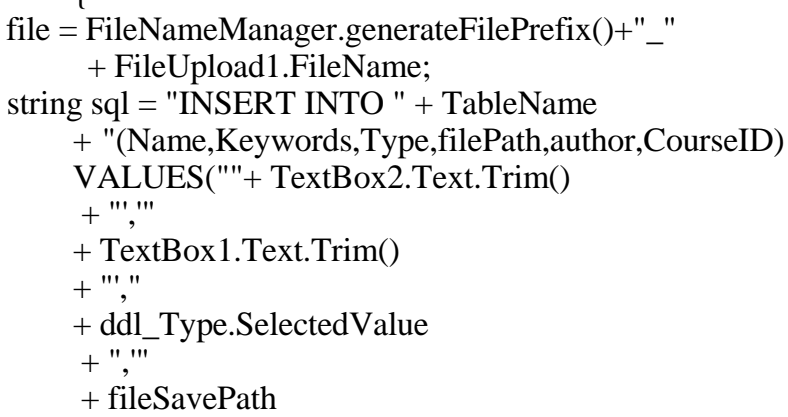




$$
\begin{aligned}
& \text { + file } \\
& \text { + "',"' } \\
& \text { + Session["UID"].ToString() } \\
& \text { + "'," } \\
& \text { + ddl_course.SelectedValue +")"; } \\
& \text { dm.executeUpdate(sql); }
\end{aligned}
$$

lb_Msg.Text = "your file has upload, thank you!";

$$
\text { V. USING THE TEMPLATE }
$$

Based on the campus network, net technology, and SQL server database, the multimedia library provides various functions, such as modification, deleting, uploading, and retrieval of the materials. With excellent openness and scalability, it shares the library freely throughout the campus. So during the courseware making, it will be more convenient for the teachers and students to search, preview, upload, or download the material, etc.

\section{REFERENCES}

[1] Xiang, C.C, "Problems and countermeasures of university teachers' multimedia teaching," Education Exploration, vol. 3, pp. 41-42, 2013.

[2] Wang, C, "Discussion on construction of university teaching material," Education and Vocation, vol.35, pp. 191-192, 2014.

[3] Wang, J.D \& Zhu, Y, "Design and implementation of multimedia library resource management system," Enterprise Science and Technology \& Development, vol.1, pp. 31-32, 2014.

[4] Wang, Y.F, Zhang, L.J \& Yang, G.H, "Design of multimedia teaching material library based on WEB," Network Wealth, vlo.5, pp.153-157, 2008. 